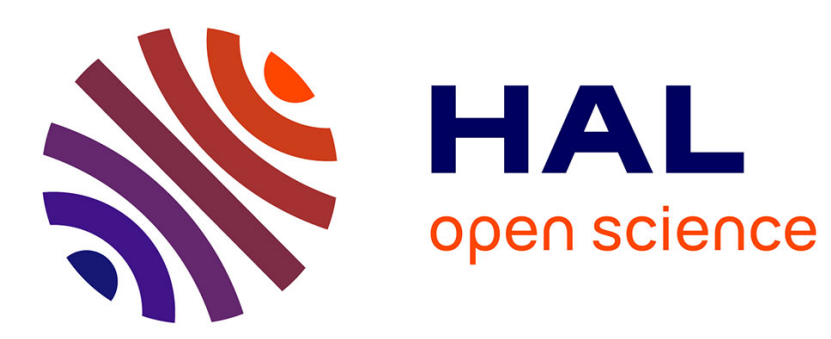

\title{
Contributions to the Problem of High-Gain Observer Design for Hyperbolic Systems
}

\author{
Constantinos Kitsos, Gildas Besancon, Christophe Prieur
}

\section{To cite this version:}

Constantinos Kitsos, Gildas Besancon, Christophe Prieur. Contributions to the Problem of High-Gain Observer Design for Hyperbolic Systems. Jiang ZP., Prieur C., Astolfi A. (eds). Trends in Nonlinear and Adaptive Control, A Tribute to Laurent Praly for his 65th Birthday, 488, Springer, pp.109-134, 2022, Lecture Notes in Control and Information Sciences, 978-3-030-74627-8. 10.1007/978-3-03074628-5_5. hal-03349184

\section{HAL Id: hal-03349184 \\ https://hal.science/hal-03349184}

Submitted on 20 Sep 2021

HAL is a multi-disciplinary open access archive for the deposit and dissemination of scientific research documents, whether they are published or not. The documents may come from teaching and research institutions in France or abroad, or from public or private research centers.
L'archive ouverte pluridisciplinaire HAL, est destinée au dépôt et à la diffusion de documents scientifiques de niveau recherche, publiés ou non, émanant des établissements d'enseignement et de recherche français ou étrangers, des laboratoires publics ou privés. 


\title{
Contributions to the Problem of High-Gain Observer Design for Hyperbolic Systems
}

\author{
Constantinos Kitsos, Gildas Besançon, and Christophe Prieur
}

\begin{abstract}
This chapter proposes some non-trivial extensions of the classical highgain observer designs for finite-dimensional nonlinear systems to some classes of infinite-dimensional systems, written as triangular systems of coupled first-order hyperbolic partial differential equations (PDEs), where an observation of one coordinate of the state only is considered as the system's output. These forms may include some epidemic models and tubular chemical reactors. To deal with this problem, depending on the number of distinct velocities of the hyperbolic system, direct and indirect observer designs are proposed. We first show intuitively how direct observer design can be applied to quasilinear partial integro-differential hyperbolic systems of balance laws with a single velocity, as a natural extension of the finitedimensional case. We then introduce an indirect approach for systems with distinct velocities (up to three velocities), where an infinite-dimensional state transformation first maps the system into a suitable systems of Partial Differential Equations (PDEs) and the convergence of the observer is subsequently exhibited in appropriate norms. This indirect approach leads to the use of spatial derivatives of the output in the observer dynamics.
\end{abstract}

Constantinos Kitsos

was with Univ. Grenoble Alpes, CNRS, Grenoble INP ${ }^{\circ}$, GIPSA-lab, 38000 Grenoble, France and is currently with LAAS-CNRS, Univ. of Toulouse, CNRS, Toulouse, France, e-mail: constantinos.kitsos@laas.fr

Gildas Besançon and Christophe Prieur

are with Univ. Grenoble Alpes, CNRS, Grenoble INP ${ }^{\circ}$, GIPSA-lab, 38000 Grenoble, France, email: \{gildas.besancon, christophe.prieur \}@ grenoble-inp.fr.

- Institute of Engineering Univ. Grenoble Alpes 


\section{Dedication}

Control Theory owes a lot to Laurent Praly and his scientific legacy has inspired the development of various new areas in Control Theory. His long-term and still ongoing contributions in adaptive robust control and stabilization, forwarding and backstepping methods, nonlinear observers and output feedback control are deeply motivating, and it is a pleasure to propose the present chapter as a tribute to his work, the quality of presentation of his results, the rigor in his theorems, and his continuous pursuit for the most possible generality of the approaches.

\section{Introduction}

Our chapter deals with solutions to a problem of High-Gain Observer design $(\mathrm{H}-$ GODP) for hyperbolic systems. This problem for the case of finite-dimensional systems has already been addressed (see [12], [13]) and has gained significant consideration in the last decades [18], [17]. These observers rely on a tuning parameter (gain), chosen large enough, in order to compensate for the nonlinear terms and ensure arbitrary convergence rate. This chapter aims at presenting some extensions of this design to infinite-dimensional systems, namely hyperbolic systems of balance laws, obeying to some triangular structure, similarly to the observability form in the finite dimensions, see [4], while considering as measurement a part of the state only. There exist some studies on observer design for infinite-dimensional systems in the literature, mainly considering the full state vector on the boundaries as measurement. Amongst others, one can refer to [11], [14], [2], and [29] for Lyapunov-based analysis and backstepping, and to [26] for optimization methods. The case of state estimation for nonlinear infinite-dimensional systems, which is significantly more complicated, has been addressed in [30], [5], [25], [28], [15], [6]. Unlike these approaches, the present chapter provides solutions to this H-GODP, where a part of the state is fully unknown (including at the boundaries). The known part is however distributed and the explored observers strongly rely on high gain, extending techniques and performances of finite-dimensional cases.

In general, the problem of control/observer design with reduced number of controls/observations, less than the number of the states, is a difficult problem. To the best of our knowledge, observer design for systems with reduced number of observations, whose error equations cannot achieve dissipativity in their boundary conditions, has not been considered. Somehow dual problems of controllability for cascade systems of PDEs with reduced number of internal controls have already been considered (see in particular [1]). In [24], observability for coupled systems of linear PDEs with reduced number of observations is studied. In this work, we reveal some links to these works, coming from our assumption on stronger regularity of the system. Additionally to this, for hyperbolic systems, arbitrary convergence, a feature of high-gain observers, would be desirable, since the boundary observers proposed in the literature, for instance [6], experience a limitation with respect to 
convergence speed (transport phenomena). The minimum-time control problem, see for instance [10], suggests that a faster observer than a boundary one, would be desirable in some cases. While dealing with the H-GODP in infinite dimensions, the assumed triangularity of the source terms, similarly to the finite dimension case, is not enough and several difficulties come from the properties of the hyperbolic operator. This might not allow designs for any large number of states. Also, the presence of nonlocal terms in the dynamics, the generality of the boundary conditions, and types of nonlinearities increase the complexity of the design.

The main contribution here is the proof of solvability of the H-GODP first for $n \times n$ quasilinear hyperbolic triangular systems with nonlocal terms, i.e., systems of partial integrodifferential equations (PIDEs), considering only a single velocity function. Then, in the case of distinct velocities, the nonexistence of diagonal Lyapunov functionals that would result in the proof of the observer convergence, leads us to adopt an indirect strategy for the case of $2 \times 2$ and $3 \times 3$ systems. For this, we introduce a nonlinear infinite-dimensional state transformation, in order to map initial system into a new system of PDEs. The problem comes from the lack of a commutative property, yet needed in the Lyapunov stability analysis. Note that constraints on the source term can be found in some studies of stability problems as in [3], [9] and in [27], which allow a similar commutation, while this is not the case here. The methodology proposed here requires stronger regularity for system's dynamics and then ouput's spatial derivatives up to order 2 are injected in the highgain observer dynamics additionally to the classical output correction terms. The presence of nonlinearities in the velocity functions, which might also have nonlocal nature, the presence of nonlocal and nonlinear components in the source terms, and the generality of the couplings on the boundaries are treated explicitly. The proposed approach relies on Lyapunov analysis for spaces of higher regularity and the introduction of an infinite-dimensional state transformation. The direct observer design has already partially appeared in [20], without considering velocity functions of nonlocal nature. The indirect one is inspired by our previous work for semilinear parabolic systems [21] and for the case of quasilinear strictly hyperbolic systems, as the ones considered here, it has not appeared before.

In Section 2, we introduce the considered system, some examples from epidemics and chemical reactions and then the main observer design problem H-GODP, along with its complications and some proposed solutions. In Section 3, we present a direct approach for the H-GODP for a hyperbolic system with one velocity via a Lyapunov-based methodology. In Section 4, we show indirect solvability of the HGODP for systems with distinct velocities, via a suitable infinite-dimensional state transformation, which maps system into appropriate target systems for observer design.

Notation: For a given $w$ in $\mathbb{R}^{n},|w|$ denotes its usual Euclidean norm. For a given constant matrix $A$ in $\mathbb{R}^{n \times n}, A^{\top}$ denotes its transpose, $|A|:=\sup \{|A w|,|w|=1\}$ is its induced norm and $\operatorname{Sym}(A)=\frac{A+A^{\top}}{2}$ stands for its symmetric part. By eig $(A)$ we denote the minimum eigenvalue of a symmetric matrix $A$. By $I_{n}$ we denote the identity matrix of dimension $n$. For given $\xi:[0,+\infty) \times[0, L] \rightarrow \mathbb{R}^{n}$ and time $t \geq 0$ we use the notation $\xi(t)(x):=\xi(t, x)$, for all $x$ in $[0, L]$ to refer to the profile at certain 
time and with $\xi_{t}$ or $\partial_{t} \xi$ (resp. $\xi_{x}$ or $\partial_{x} \xi$ ) we refer to its partial derivative with respect to $t$ (resp. $x$ ). By $\mathrm{d}_{t}$ (resp. $\mathrm{d}_{x}$ ) we refer to the total derivative with respect to $t$ (resp. $x$ ). For a continuous $\left(C^{0}\right)$ map $[0, L] \ni x \mapsto \xi(x) \in \mathbb{R}^{n}$ we adopt the notation $\|\xi\|_{0}:=\max \{|\xi(x)|, x \in[0, L]\}$ for its sup-norm. If this mapping is $q$ - times continuously differentiable $\left(C^{q}\right)$, we adopt the notation $\|\xi\|_{q}:=\sum_{i=0}^{q}\left\|\partial_{x}^{i} \xi\right\|_{0}$ for the $q$-norm. We use the difference operator given by $\Delta_{\hat{\xi}}[\mathscr{F}](\xi):=\mathscr{F}[\hat{\xi}]-\mathscr{F}[\xi]$, parametrized by $\hat{\xi}$, where $\mathscr{F}$ denotes any chosen operator acting on $\xi$. By $D f$ we denote the Jacobian of a differentiable mapping $\mathbb{R}^{n} \ni u \mapsto f(u) \in \mathbb{R}^{m}$. For a Fréchet differentiable mapping $\mathscr{F}$, by $\langle D F[u], h\rangle$ we denote its Fréchet derivative w.r.t. $u$ acting on $h$. For a locally Lipschitz mapping $\mathscr{F}, \mathscr{F} \in \operatorname{Lip}_{\text {loc }}(\mathscr{X},\|\cdot\| \mathscr{X})$ means that for every $R>0$, there exists $L_{R}>0$, such that for every $w, \hat{w} \in \mathscr{X}$, with $\|w\|_{\mathscr{X}},\|\hat{w}\|_{\mathscr{X}} \leq R$, it holds $\|\mathscr{F}[w]-\mathscr{F}[\hat{w}]\|_{\mathscr{X}} \leq L_{R}\|w-\hat{w}\|_{\mathscr{X}}$. For a globally Lipschitz mapping $\mathscr{F}$, i.e., $\mathscr{F} \in \operatorname{Lip}\left(\mathscr{X},\|\cdot\|_{\mathscr{X}}\right)$, the previous holds for all $w, \hat{w} \in \mathscr{X}$. By $\operatorname{sgn}(x)$ we denote the signum function $\operatorname{sgn}(x)=\frac{\mathrm{d}}{\mathrm{d} x}|x|$, when $x \neq 0$, with $\operatorname{sgn}(0)=0$.

\section{Problem Description and Solutions}

In this section we introduce the hyperbolic system written in a triangular form, which allows the observer design proposed in this chapter. It might be quasilinear and contain also both velocity functions of nonlocal nature and nonlocal source terms, making it a system of PIDEs. We illustrate some examples of systems having this triangular form and then we introduce the main observer problem and its solutions.

\subsection{Triangular Form for Observer Design}

We are concerned with one-dimensional hyperbolic systems of balance laws, described by the following equations on a strip $\Pi:=[0,+\infty) \times[0, L]$

$$
\xi_{t}(t, x)+\Lambda\left[\xi_{1}(t)\right](x) \xi_{x}(t, x)=A \xi(t, x)+\mathscr{F}[\xi(t)](x),
$$

where $\xi=\left(\xi_{1} \ldots \xi_{n}\right)^{\top}$. The matrix function $\Lambda[\cdot]$ contains the velocity functions of the balance law, each of which assumed strictly positive, and is diagonal of the form

$$
\Lambda\left[\xi_{1}\right]:=\operatorname{diag}\left\{\lambda_{1}\left[\xi_{1}\right], \ldots, \lambda_{n}\left[\xi_{1}\right]\right\} .
$$

We assume a specific structure of the source terms, which provides an internal coupling of the $n$ equations in a triangular fashion. More specifically, matrix $A$ contains $1 \mathrm{~s}$ on its sup-diagonal and 0 s elsewhere, i.e., it performs the operation

$$
A \xi=\left(\begin{array}{lllll}
\xi_{2} & \xi_{3} & \ldots & \xi_{n} & 0
\end{array}\right)^{\top}
$$


and the nonlinear source term $\mathscr{F}(\cdot)$ has the following form

$$
\mathscr{F}(\xi)=\left(\mathscr{F}_{1}\left[\xi_{1}\right] \mathscr{F}_{2}\left[\xi_{1}, \xi_{2}\right] \ldots \mathscr{F}_{n}\left[\xi_{1}, \ldots, \xi_{n}\right]\right)^{\top}
$$

We assume that mappings $\Lambda$ might include local terms of the form $\lambda_{i}\left(\xi_{1}(t, x)\right)$ or nonlocal terms of the form $\lambda_{i}\left(\int_{0}^{x} \xi_{1}(t, s) \mathrm{d} s\right)$ or $\int_{0}^{x} \lambda_{i}\left(\xi_{1}(t, s)\right) \mathrm{d} s$ for instance. Same for the nonlinear source term that might include local terms of the form $f(x, \xi(t, x))$, integral terms of Volterra type of the form $\int_{0}^{x} f(s, \xi(t, s)) \mathrm{d} s$, and possibly boundary terms of the form $f(\xi(t, l))$, with $l=0, L$.

Consider, also, a distributed measurement, available at the output, written as follows

$$
y(t, x)=C \xi(t, x)
$$

where

$$
C=\left(\begin{array}{llll}
1 & 0 & \cdots & 0
\end{array}\right)
$$

To complete the definition of the class of systems, let us consider initial condition (in general unknown) $\xi^{0}$ and boundary conditions as follows

$$
\begin{aligned}
& \xi(0, x)=\xi^{0}(x), x \in[0, L], \\
& \xi(t, 0)=\mathscr{H}(\xi(t, L)), t \in[0,+\infty),
\end{aligned}
$$

where $\mathscr{H}$ is a nonlinear mapping coupling the incoming with the outgoing information on the boundaries.

More about the regularity of the dynamics and the properties of the system will be provided in the forthcoming sections. Note that the above system has the same structure up to the hyperbolic operator as the one considered in the finite-dimensional nonlinear triangular systems, appropriate for observer designs, see [17].

We provide here some examples of dynamic phenomena coming from epidemiology and chemical reactions, that can be described by triangular systems of hyperbolic PDEs, as the ones given above. Note that some distributed Lotka-Volterra systems might also take this triangular form, as it was shown in [21], but obeying parabolic equations.

\section{- SIR Epidemic Models}

For infectious diseases, a fundamental model was formulated by Kermack and McKendrick (see [3, Chapter 1] for more details). In this model, population is classified into three groups: (i) the individuals who are uninfected and susceptible $(S)$ of catching the disease, (ii) the individuals who are infected $(I)$ by the concerned pathogen, (iii) the recovered $(R)$ individuals who have acquired a permanent immunity to the disease. Assuming that the age of patients is taken into account, $S(t, x), I(t, x), R(t, x)$ represent the age distribution of the population of each group at time $t$. As a result, the integral from $x_{1}$ to $x_{2}$ of $S, I$ and $R$ is the number of individuals of each group with ages between $x_{1}$ and $x_{2}$. 
The dynamics of the disease propagation in the population are then described by the following set of hyperbolic partial integro-differential equations on $\Pi$

$$
\begin{aligned}
& S_{t}(t, x)+S_{x}(t, x)+\mu(x) S(t, x)+\mathscr{G}[S(t), I(t)](x)=0, \\
& I_{t}(t, x)+I_{x}(t, x)+(\gamma(x)+\mu(x)) I(t, x)-\mathscr{G}[S(t), I(t)](x)=0, \\
& R_{t}(t, x)+R_{x}(t, x)+\mu(x) R(t, x)-\gamma(x) I(t, x)=0,
\end{aligned}
$$

where $\mathscr{G}[S(t), I(t)](x):=\beta(x) S(t, x) \int_{0}^{L} I(t, s) \mathrm{d} s$ stands for the disease transmission rate by contact between susceptible and infected individuals, which is assumed to be proportional to the sizes of both groups, with $\beta(x)>0$ being the age-dependent transmission coefficient between all infected individuals and susceptibles having age $x$. The maximal life duration in the considered population is denoted by $L$ and, thus, $S(t, L)=I(t, L)=R(t, L)=0$. Parameter $\mu(x)>0$ denotes the natural age-dependent per capita death rate in the population and $\gamma(x)>0$ is the age-dependent rate at which infected individuals recover from the disease. We also assume some boundary conditions of the form $S(t, 0)=B(t), I(t, 0)=0, R(t, 0)=0$, where $B(t)$ stands for the inflow of newborn individuals in the susceptible part of the population at time $t$. Assume that we are able to measure the number of people in the group $R$ of recovered patients between ages 0 and $x$, for every age $x \in[0, L]$ and time $t \geq 0$, i.e., system's output is given by the quantity $\int_{0}^{x} R(t, s) \mathrm{d} s$.

System (3) is written in the form (1a)-(1b)-(2) by applying a nonlocal transformation of the following form

$$
\begin{aligned}
& \xi_{1}(t, x)=\int_{0}^{x} R(t, s) \mathrm{d} s, \\
& \xi_{2}(t, x)=\int_{0}^{x} \gamma(s) I(t, s) \mathrm{d} s, \\
& \xi_{3}(t, x)=\int_{0}^{x} \beta(s) \gamma(s) S(t, s) \mathrm{d} s \int_{0}^{L} I(t, s) \mathrm{d} s .
\end{aligned}
$$

Then, in the new coordinates, system is written in the general form (1a) we considered here, with constant velocities, namely, $\Lambda\left[\xi_{1}\right]=I_{n}$ and with its nonlinear source term having the form $\mathscr{F}[\xi(t)](x):=\mathscr{F}(x, \xi(t)(x))$, containing also nonlinear nonlocal terms, more explicitly, some integral terms of Volterra type and boundary terms. For the exact form of these mappings that we derive after the transformation (4), the reader can refer to [20].

Such a problem, where the hyperbolic operator has a single velocity, is investigated in Section 3, which allows a direct observer design. Note also that due to the nonlocal nature of the transformation (4), one needs to prove the convergence of a candidate observer for system in the new coordinates $\xi$ in the 1-spatial norm (and not in the sup-spatial norm), in order to be able to return to the original coordinates of the SIR model. 


\section{- Tubular chemical reactors}

Control and observer designs for chemical reactors in the sense of distributed parameter systems have been widely investigated, see for instance [7]. We present here a model of a parallel plug flow chemical reactor (see [3, Chap 5.1]). A plug flow chemical reactor is a tubular reactor where a liquid reaction mixture circulates. The reaction proceeds as the reactants travel through the reactor. We consider the case of a horizontal reactor, where a simple mono-molecular reaction takes place between $A$ and $B$, where $A$ is the reactant species and $B$ is the desired product. The reaction is supposed to be exothermic and a jacket is used to cool the reactor. The cooling fluid flows around the wall of the tubular reactor. The dynamics are described by the following hyperbolic equations on $\Pi$

$$
\begin{aligned}
& \partial_{t} T_{c}+V_{c} \partial_{x} T_{c}-k_{0}\left(T c-T_{r}\right)=0, \\
& \partial_{t} T_{r}+V_{r} \partial_{x} T_{r}+k_{0}\left(T_{c}-T_{r}\right)-k_{1} r\left(T_{r}, C_{A}\right)=0, \\
& \partial_{t} C_{A}+V_{r} \partial_{x} C_{A}+r\left(T_{r}, C_{A}\right)=0,
\end{aligned}
$$

where $V_{c}$ is the coolant velocity in the jacket, $V_{r}$ is the reactive fluid velocity in the reactor, $k_{0}$ and $k_{1}$ are some positive constants, $T_{c}(t, x)$ is the coolant temperature, $T_{r}(t, x)$ is the reactor temperature, $C_{A}(t, x)$ is the concentration of the chemical $A$ in the reaction medium. The function $r\left(T_{r}, C_{A}\right)$ stands for the reaction rate and is given by $r\left(T_{r}, C_{A}\right):=\left((a+b) C_{A}-b C_{A}^{\text {in }}\right) \exp \left(-\frac{E}{R T_{r}}\right)$ where we have assumed that the sum of concentrations $C_{A}+C_{B}$ is constant, equal to $C_{A}(t, x)+C_{B}(t, x)=C_{A}^{\text {in }}$, as it is simply described by a delay equation. Also, $a, b$ are rate constants, $C_{A}^{\text {in }}$ is the concentration on the left endpoint, $E$ is the activation energy and $R$ is the Boltzmann constant. We consider boundary conditions of the form $T_{r}(t, 0)=T_{r}^{\text {in }}, T_{c}(t, 0)=T_{c}^{\text {in }}, C_{A}(t, 0)=C_{A}^{\text {in }}, C_{B}(t, 0)=0$. Assuming that the measured quantity is the coolant temperature $T_{c}$, we can transform system (5) into a form as (1a)-(1b)-(2) by applying the invertible transformation

$$
\xi_{1}=T_{c}, \quad \xi_{2}=T_{r}, \quad \xi_{3}=k_{1}\left((a+b) C_{A}-b C_{A}^{\text {in }}\right) \exp \left(-\frac{E}{R T_{r}}\right) .
$$

In this example, the hyperbolic operator has distinct velocities. Such a problem is investigated in Section 4.

\subsection{The High-Gain Observer Design Problem}

We present here the main problem this chapter deals with and some proposed solutions.

Definition 1. (H-GODP) The High-Gain Observer Design Problem is solvable for a system written in the form (1a)-(2) with output (1b), while output's spatial derivatives of order at most $n-1$ might also be available, if there exists a well-posed 
observer system of PDEs, which estimates the state of initial system with a convergence speed that can be arbitrarily tuned via a single parameter (high-gain constant) $\theta$. More precisely, for every $\kappa>0$, there exists $\theta_{0}>1$, such that for every $\theta \geq \theta_{0}$, solutions to (1a)-(2) satisfy

$$
\|\hat{\xi}(t, \cdot)-\xi(t, \cdot)\|_{\mathscr{X}_{1}} \leq \ell e^{-\kappa t}\left\|\hat{\xi}^{0}(\cdot)-\xi^{0}(\cdot)\right\|_{\mathscr{X}_{2}}
$$

for some $\ell>0$ polynomial in $\theta$, where $\hat{\xi}, \hat{\xi}^{0}$ represent the observer state and its initial condition respectively and by $\mathscr{X}_{1}, \mathscr{X}_{2}$ we denote some function spaces, whose accurate choice depends on the number of distinct velocities.

A feature of this observer design problem is the considered internal measurement of part of the state, without any other knowledge on the other states. Furthermore, another feature indicated in the H-GODP definition, is a required stronger regularity of the solutions to the initial systems, since the observer dynamics may include spatial derivatives of the output. This requirement reveals some links to previous studies on internal controllabity for cascade systems with reduced number of controls, see [1]. We note here that, although boundary observers with the full-state measurement are preferred for practical reasons, see for instance [6], in the present formulation distributed measurement of part of the state might be available in many cases, for instance thermal cameras for chemical reactors or approximations with distributed measurements within the domain. Additionally, the required spatial derivatives of the output can be available in real-time, since they follow from causal measurements, contrary to the time-derivatives of the output, which are strictly not included in observer designs, as the knowledge of them is non-causal. Although this requirement of the availability of space derivatives of the output might seem restrictive, approximations via kernel convolutions might be an alternative realization.

Remark 1. Solvability of the H-GODP suggests that a high-gain observer would be arbitrarily fast, without any limitation in the convergence speed. H-GODP is not solvable in case of boundary measurement, instead of internal measurement as in (1b). First, arbitrary convergence condition would not be fulfilled, since a boundary observer for hyperbolic systems would experience a limitation with respect to convergence speed. The rate of convergence is limited by a minimal observation time which depends on the size of the domain and the velocities in that case (see [23] for minimum time of observability due to transport phenomena). Second, following a boundary observer design methodology as in [6], in the presence of a general form of boundary conditions, where a nonlinear law couples the incoming with the outgoing information on the boundaries, boundary measurement of the whole state vector would be required, instead of just the first state, for the boundary observer to be feasible. In [8], control design is achieved for a $2 \times 2$ hyperbolic system with some specific boundary conditions, via boundary control on one end of only one state. Here, however, where we consider the dual problem of observer design with one observation, such an approach would not be feasible, because for general boundary conditions, by just one observation we cannot achieve a dissipativity of the bound- 
ary conditions as in this work, which would lead to stability of the observation error system (see [3] about linking dissipativity of boundary conditions with stability).

The main problem appearing when dealing with the solvability of the H-GODP, comes from the hyperbolic operator. More particularly, the form and also the domain of the hyperbolic operator might be general, including distinct velocities and also very general couplings of the incoming with the outgoing information on the boundaries. In stability analysis of hyperbolic systems, diagonal Lyapunov functionals are usually chosen, see [3], since in taking its time-derivative, an integration by parts is required, which can be simplified if the Lyapunov matrix commutes with the diagonal matrix of the velocities $\Lambda[\cdot]$. In our case, the Lyapunov matrix cannot be diagonal, since it shall solve a quadratic Lyapunov equation for the non-diagonally stabilizable matrix $A$. Section 3 deals with a solution to this H-GODP for a system with one velocity, where an extension from finite dimensions is direct, since the aforementioned commutative property is met. In Section 4 we elaborate an indirect design, where the general hyperbolic operator of distinct velocities is decomposed into a new hyperbolic operator with one velocity plus some mappings acting only on the measured first state, and a bilinear triangular mapping between the measured first state and the second one. Additionally to these complications, note that for the solvability of the H-GODP, difficulties also come from the presence of nonlocal terms, which require stability proof in the sup-norm, and also, from the quasiliniearity of the system, i.e., the dependence of $\Lambda[\cdot]$ on the state.

\section{Observer Design for Systems with a Single Velocity}

In this section, we show the solvability of the H-GODP for a system with a single velocity, which constitutes a direct extension of observer design in finite dimensions.

\subsection{Problem Statement and Requirements}

Consider the general hyperbolic system (1a)-(2), with output (1b) and with the same triangularity of its mappings given therein. We assume in this section that system has only one velocity, i.e., matrix of velocities is of the form

$$
\Lambda\left[\xi_{1}\right]:=\lambda\left[\xi_{1}\right] I_{n}
$$

where velocity $\lambda: C^{1}([0, L] ; \mathbb{R}) \rightarrow C^{1}([0, L] ; \mathbb{R})$ is Fréchet differentiable, possibly nonlocal, and positive (namely, $\lambda[y]>0$, for all $y \in C^{0}([0, L] ; \mathbb{R})$ (nonlocal case), or $y \in \mathbb{R}$ (local case)), and nonlinear mapping $\mathscr{F}[\xi(t)](x):=\mathscr{F}(x, \xi(t)(x))$, with $\mathscr{F}:[0, L] \times C^{1}\left([0, L] ; \mathbb{R}^{n}\right) \rightarrow C^{1}\left([0, L] ; \mathbb{R}^{n}\right)$ is continuously differentiable with respect to its first argument and Fréchet differentiable with respect to its second argument. It can possibly contain nonlocal terms (integral terms of Volterra type 
and boundary terms). We further assume that $D \mathscr{F} \in \operatorname{Lip}_{\text {loc }}\left(C^{0}\left([0, L] ; \mathbb{R}^{n}\right),\|\cdot\|_{0}\right)$. Also, initial condition $\xi^{0} \in C^{1}\left([0, L] ; \mathbb{R}^{n}\right)$ satisfies zero-order and one-order compatibility conditions (see [3, App. B] for precise definition of compatibility conditions) and the nonlinear mapping $\mathscr{H}$ coupling the incoming with the outgoing information is in $C^{1}\left(\mathbb{R}^{n} ; \mathbb{R}^{n}\right)$, while its gradient is locally Lipschitz continuous, i.e., D $\mathscr{H} \in \operatorname{Lip}_{\text {loc }}\left(\mathbb{R}^{n},|\cdot|\right)$.

The assumption that follows is essential to assert the well-posedness of the considered system, along with an observer design requirement of forward completeness. Furthermore, it imposes global boundedness of classical solutions in the 1-norm. The latter requirement is due to the quasi-linearity of the system (the dependence of $\lambda$ on $\xi_{1}$ ) and can be dropped for the case of semilinear systems, but then a stronger assumption on the nonlinear source terms would be imposed instead. For more detailed presentation of the nature of the following assumption, the reader can refer to [3] and references therein, where sufficient conditions for the well-posedness and existence of classical solutions for hyperbolic systems are given. In the case of nonlocal conservation laws, i.e., where velocity $\lambda: C^{0}([0, L] ; \mathbb{R}) \rightarrow C^{1}([0, L] ; \mathbb{R})$ might be of the form $\lambda\left[\xi_{1}(t)\right](x):=\lambda\left(\int_{0}^{x} \xi_{1}(t, s) \mathrm{d} s\right)$, this assumption can be met more easily, see for instance [16] and other works of these authors.

Assumption 1 Consider a set $\mathscr{M} \subset C^{1}([0, L] ; \mathbb{R})$ nonempty and bounded, consisting of functions satisfying zero-order and one-order compatibility conditions for problem (1a)-(2). Then for any initial condition $\xi^{0}$ in $\mathscr{M}$, problem (1a)-(2) admits a unique classical solution in $C^{1}\left([0,+\infty) \times[0, L] ; \mathbb{R}^{n}\right)$. Moreover, there exists $\delta>0$, such that for all $\xi^{0}$ in $\mathscr{M}$, we have $\xi \in \mathscr{B}_{\delta}^{1}:=\left\{u \in C^{1}\left([0, L] ; \mathbb{R}^{n}\right):\|u\|_{1} \leq \delta\right\}$.

With these assumptions, we are in a position to introduce our candidate observer dynamics and its boundary conditions on $\Pi$ for system (1)-(2), as follows

$$
\begin{aligned}
\hat{\xi}_{t}(t, x)+\lambda[y(t)](x) \hat{\xi}_{x}(t, x)= & A \hat{\xi}(t, x)+\mathscr{F}\left[s_{\delta}(\hat{\xi}(t))\right](x) \\
& -\Theta K(y(t, x)-C \hat{\xi}(t, x)), \\
\hat{\xi}(t, 0)= & \mathscr{H}\left(s_{\delta}(\hat{\xi}(t, L))\right)
\end{aligned}
$$

where the function $s_{\delta}: \mathbb{R}^{n} \ni \zeta \mapsto s_{\delta}(\zeta)=\left(s_{\delta}^{1}\left(\zeta_{1}\right), \cdots, s_{\delta}^{n}\left(\zeta_{n}\right)\right)$ is parametrized by $\delta$ (given in Assumption 1) and satisfies the following properties

1. it is uniformly bounded and continuously differentiable;

2. its first derivative is uniformly bounded;

3. its derivative function $D s_{\delta}(\cdot)$ is in $\operatorname{Lip}\left(\mathbb{R}^{n},|\cdot|\right)$;

4. for every $\delta>0$ and $v, w$ in $\mathbb{R}^{n}$, such that $|w| \leq \delta$, there exists $\omega_{\delta}>0$, such that the following inequality is satisfied

$$
\left|s_{\delta}(v)-w\right| \leq \omega_{\delta}|v-w| .
$$

Note that a saturation-like function of the form 


$$
s_{\delta}^{i}\left(\zeta_{i}\right)= \begin{cases}\zeta_{i}, & \left|\zeta_{i}\right| \leq \delta \\ \operatorname{sgn}\left(\zeta_{i}\right)\left(\left(\left|\zeta_{i}\right|-\delta\right) e^{-\left|\zeta_{i}\right|+\delta}+\delta\right), & \left|\zeta_{i}\right|>\delta\end{cases}
$$

satisfies all properties and, particularly, (8) with $\omega_{\delta}=\sqrt{n} \max \left\{1, e^{-1}+\delta\right\}$ and with Lipschitz constant of $D s_{\delta}(\cdot)$ equal to $\sqrt{n} e^{-3}$.

Also, $\Theta$, appearing in the output correction term of the observer, is a diagonal matrix given by

$$
\Theta:=\operatorname{diag}\left\{\theta, \theta^{2}, \ldots, \theta^{n}\right\},
$$

where $\theta>1$ is the candidate high-gain constant of the observer, which will be selected precisely later, and $K \in \mathbb{R}^{n}$ is chosen such that $A+K C$ is Hurwitz (we can always find such a $K$, due to the observability of the pair $(A, C)$ ). Note that for such a $K$, one can find a symmetric and positive definite $n \times n$ matrix $P$ satisfying a quadratic Lyapunov equation of the following form

$$
2 \operatorname{Sym}(P(A+K C))=-I_{n} .
$$

Let us remark that $P$ satisfying (11) cannot be diagonal, since matrix $A$ fails by its definition to be a diagonally stabilizable matrix. The matrix $P$ will be used as the Lyapunov matrix in the Lyapunov functional used in the proof of the observer convergence. However, in stability analysis of general hyperbolic systems, see for instance [3], the chosen Lyapunov functionals are diagonal, in order to commute with the matrix of the velocities. In the present case, we assume only one velocity and, thus, we do not need that $P$ is diagonal.

\subsection{Direct Solvability of the H-GODP}

We are in a position to present our main result on the solvability of the H-GODP via observer system (7).

Theorem 1. Consider system (1a)-(2), with a single velocity $\lambda$ and output (1b), and suppose that Assumption 1 holds for initial condition $\xi^{0}$ in $\mathscr{M}$. Let also $K$ in $\mathbb{R}^{n}$, chosen in such a way that $A+K C$ is Hurwitz. Then, the H-GODP for system (1a) - (2) is solvable by system (7) for $\theta>1$ as a high gain and initial condition $\hat{\xi}^{0}$ in $C^{1}\left([0, L] ; \mathbb{R}^{n}\right)$, with $\hat{\xi}^{0}(x)=\hat{\xi}(0, x)$, satisfying zero-order and one-order compatibility conditions. More precisely, for every $\kappa>0$, there exists $\theta_{0} \geq 1$, such that for every $\theta>\theta_{0}$, the following inequality holds

$$
\|\hat{\xi}(t, \cdot)-\xi(t, \cdot)\|_{1} \leq \ell e^{-\kappa t}\left\|\hat{\xi}^{0}(\cdot)-\xi^{0}(\cdot)\right\|_{1}, \forall t \geq 0
$$

for some $\ell>1$, polynomial in $\theta$.

Note that Theorem 1 shows solvability of the H-GODP of Definition 1 with no use of spatial derivatives of the output. This is the reason why we call this approach 
direct. This result slightly generalizes [20] and [19] in the sense that it considers also the case of a velocity function of nonlocal nature.

Proof. Prior to the observer convergence, existence and uniquenes of global classical solutions to the observer system, which is a semilinar hyperbolic system with possibly nonlocal terms, must be proven. The reader can refer to [22, Theorem 2.1] and, similarly to that work, we can follow a fixed-point methodology, taking into account the sufficient regularity of the dynamics, the global boundedness of system's solutions (and, thus of the output $y$ ) coming from Assumption 1, and also the fact that the nonlinearities appearing in the observer system are globally Lipschitz. More details can be found in [20, Appendix A].

Consider the following linearly transformed observer error

$$
\varepsilon:=\Theta^{-1}(\hat{\xi}-\xi)
$$

for which we derive the following hyperbolic equations on $\Pi$

$$
\begin{aligned}
\varepsilon_{t}(t, x)+\lambda[y(t)](x) \varepsilon_{x}(t, x)= & \theta(A+K C) \varepsilon(t, x) \\
& +\Theta^{-1} \Delta_{z(t)}[\mathscr{F}](\xi(t))(x), \\
\varepsilon(t, 0)= & \Theta^{-1} \Delta_{z(t)}[\mathscr{H}](\xi(t))(L),
\end{aligned}
$$

where $z:=s_{\delta}(\hat{\xi})$. Notice that in the above internal dynamics, $\theta(A+K C) \varepsilon$ will be used as a damping term leading to the exponential stabilization of the error dynamics.

To prove exponential stability of the error system at the origin in the 1-norm, we adopt a Lyapunov approach inspired by methodologies presented in [3]. The proof is included in the Appendix. A slightly different proof for a local velocity function has appeared in [20].

Following the proof in the Appendix, the H-GODP for (1a), (1b), (2) is solved by designing an exponential in the 1-norm high-gain observer of adjustable convergence rate, dependent on the selection of $\theta$.

\section{Observer Design for Systems with Distinct Velocities}

In this section, we employ an indirect strategy, in order to show solvability of the H-GODP, when the velocities are not identical. To this end, we first map system into an appropriate system of PDEs via an introduced nonlinear infinite-dimensional state transformation, noting however that for nonlinear systems with more than three states, accompanied with more than three distinct velocities, such a state transformation-based approach is difficult to be employed. 


\subsection{System Requirements and Main Approach}

Consider again system (1)-(2), but with the restriction of up to 3 states, namely

$$
n \in\{2,3\} .
$$

To provide the properties and appropriate regularity of the dynamics of the considered system, let us first define a, roughly speaking, "index of strict hyperbolicity" as follows

$$
q:=\min \left\{i: \lambda_{i} \equiv \lambda_{j}, \forall j=i, \ldots, n\right\},
$$

where we used the equivalence relation $\lambda_{i} \equiv \lambda_{j} \Leftrightarrow \lambda_{i}\left[\xi_{1}\right]=\lambda_{j}\left[\xi_{1}\right], \forall \xi_{1} \in C^{0}([0, L] ; \mathbb{R})$. By this definition, we have $q \in\{1,2,3\}$ and in the case of a strictly hyperbolic system, we have $q=n$. The case where $q=1$ (a single velocity) was addressed in the previous section. We further define

$$
q_{0}:=\max \{1, q-1\} .
$$

We assume that velocities $\lambda_{i}: C^{q_{0}}([0, L] ; \mathbb{R}) \rightarrow C^{q_{0}}([0, L] ; \mathbb{R}), i=1, \ldots, n$ are $q_{0^{-}}$ times Fréchet differentiable and positive (namely $\lambda_{i}[y]>0$, for all $y \in C^{0}([0, L] ; \mathbb{R})$ (nonlocal case), or $y \in \mathbb{R}$ (local case)), nonlinear mapping $\mathscr{F}: C^{q_{0}}\left([0, L] ; \mathbb{R}^{n}\right) \rightarrow$ $C^{q_{0}}\left([0, L] ; \mathbb{R}^{n}\right)$ is $q_{0}$-times Fréchet differentiable and for $q_{0}=1$ we further assume that $D \mathscr{F} \in \operatorname{Lip}_{\text {loc }}\left(C^{0}\left([0, L] ; \mathbb{R}^{n}\right),\|\cdot\|_{0}\right)$. For initial condition $\xi^{0} \in C^{q_{0}}\left([0, L] ; \mathbb{R}^{n}\right)$, we assume that it satisfies compatibility conditions of order $q_{0}$ (see [3, Chapter 4.5.2] for definition of compatibility conditions of any order) and mapping $\mathscr{H}$ is of class $C^{q_{0}}\left(\mathbb{R}^{n} ; \mathbb{R}^{n}\right)$, while for $q_{0}=1$, we additionally have $D \mathscr{H} \in \operatorname{Lip} p_{\text {loc }}\left(\mathbb{R}^{n},|\cdot|\right)$.

As in the previous section, we make an assumption on existence and uniqueness of solutions of possibly stronger regularity, depending on the index of strict hyperbolicity $q$, and this assumption might be met more easily in the case of nonlocal conservation laws, see for instance [16].

Assumption 2 Consider $\mathscr{M} \subset C^{q_{0}}([0, L] ; \mathbb{R})$ nonempty and bounded, consisting of functions satisfying compatibility conditions of order $q_{0}$ for problem (1a)-(2). Then for any initial condition $\xi^{0}$ in $\mathscr{M}$, problem (1a)-(2) admits a unique solution in $C^{q_{0}}\left([0,+\infty) \times[0, L] ; \mathbb{R}^{n}\right)$. Moreover, there exists $\delta>0$, such that for all $\xi^{0}$ in $\mathscr{M}$, we have $\xi \in \mathscr{B}_{\delta}^{q_{0}}:=\left\{u \in C^{q_{0}}\left([0, L] ; \mathbb{R}^{n}\right):\|u\|_{q_{0}} \leq \delta\right\}$.

Let us define a Banach space by

$$
\mathscr{X}:=C^{q_{0}}([0, L] ; \mathbb{R}) \times C^{1}\left([0, L] ; \mathbb{R}^{n-1}\right),
$$

equipped with the norm $\|\cdot\|_{\mathscr{X}}:=\|\cdot\|_{1}$, when $n=2$ and $\|\xi\|_{\mathscr{X}}:=\left\|\xi_{1}\right\|_{q_{0}}+\left\|\xi_{2}\right\|_{1}+$ $\left\|\xi_{3}\right\|_{1}$, when $n=3$.

To deal with the generality of the considered hyperbolic operator, i.e., the presence of distinct velocities, we need to employ a different strategy than in the previous section. The problem comes from the fact that the balance laws in (1a) do 
not allow the choice of a diagonal Lyapunov functional to be used in the stability analysis of the observer error equations. A non-diagonal Lyapunov functional does not permit an integration by parts when taking its time-derivative, since the Lyapunov matrix and the matrix of velocities do not commute. To address this problem, we perform a transformation including spatial derivations of the state up to order $q-2$, in order to write the system in an appropriate form for which a Lyapunov approach is feasible. Then, for the obtained target system, we design the high-gain observer and, finally, returning to the initial coordinates, solvability of H-GODP is guaranteed. The increased difficulties with respect to the presence of distinct velocities appear in the somehow dual problems of internal controllability with reduced numbers of controls (see comments on algebraic solvability in [1]).

We shall show the existence of a nonlinear transformation $\mathscr{T}$, such that for each fixed $\xi_{1}, \mathscr{T}\left[\xi_{1}\right]:\left(\mathscr{X},\|\cdot\|_{\mathscr{X}}\right) \rightarrow\left(\mathscr{X},\|\cdot\|_{\mathscr{X}}\right)$ is bounded and injective with bounded inverse, and maps system (1a)-(2) into a target system $\zeta$, as follows

$$
\begin{gathered}
\zeta=\mathscr{T}\left[\xi_{1}\right] \xi ; \\
\text { with } \zeta_{1}=\xi_{1}
\end{gathered}
$$

Assume also that this transformation is written in the form

$$
\mathscr{T}[\cdot]=I_{n}+\tilde{\mathscr{T}}[\cdot] C,
$$

for some column operator $\tilde{\mathscr{T}}$.

The desired target system (T) of PDEs satisfies the following equations on $\Pi$

$$
(\mathrm{T})\left\{\begin{array}{l}
\zeta_{t}(t, x)+\lambda_{n}\left[\zeta_{1}(t)\right](x) \zeta_{x}(t, x)=A \zeta(t, x)+\mathscr{F}\left[\zeta(t)-\tilde{T}\left[\zeta_{1}(t)\right] C \zeta(t)\right](x) \\
+\mathscr{N}_{1}\left[\zeta_{1}(t)\right](x)+\mathscr{N}_{2}\left[\zeta_{1}(t), \zeta_{2}(t)\right](x) \\
\zeta(t, 0)=\mathscr{H}\left(\zeta(t, L)-\tilde{\mathscr{T}}\left[\zeta_{1}(t)\right] C \zeta(t)(L)\right)+\mathscr{N}_{3}\left[\zeta_{1}(t)\right](0), \\
Y(t, x)=y(t, x)=C \zeta(t, x),
\end{array}\right.
$$

with initial condition $\zeta(0, x):=\zeta^{0}(x)=\mathscr{T}\left[\xi_{1}^{0}\right] \xi^{0}(x)$, where nonlinear operators $\mathscr{N}_{1}: C^{q_{0}}([0, L] ; \mathbb{R}) \rightarrow C^{0}\left([0, L] ; \mathbb{R}^{n}\right), \mathscr{N}_{3}: C^{q_{0}}([0, L] ; \mathbb{R}) \rightarrow \mathbb{R}^{n}$ are acting on the measured state $\zeta_{1}, \mathscr{N}_{2}: C^{q_{0}}([0, L] ; \mathbb{R}) \times C^{0}([0, L] ; \mathbb{R}) \rightarrow C^{q_{0}-1}\left([0, L] ; \mathbb{R}^{n}\right)$ is a bilinear triangular mapping, to be determined in the sequel, depending on the choice of $\mathscr{T}$, and $Y$ is target system's output, which remains equal to the original system's output $y$.

In this considered target system of PDEs, we observe that system's hyperbolic operator has been decomposed into the sum of a hyperbolic one with only one velocity, the last one $\lambda_{n}$, plus a nonlinear differential operator acting only on the measured first state only $\mathscr{N}_{1}$, a bilinear mapping of the state $\mathscr{N}_{2}$, while a nonlinear differential operator $\mathscr{N}_{3}$ acting on the first state appears on the boundaries. Thus, observer design can be possible for target system (T), as we now meet the desired property of one single velocity that we imposed in the previous section, while we can simultaneously cancel the unwanted terms of the transformed system, represented by the nonlinear operators $\mathscr{N}_{1}, \mathscr{N}_{2}, \mathscr{N}_{3}$ acting on the measured state $\zeta_{1}$. The 
proposed high-gain observer for target system (T) satisfies the following equations on $\Pi$

$$
\begin{aligned}
& \hat{\zeta}_{t}(t, x)+\lambda_{n}[y(t)](x) \hat{\zeta}_{x}(t, x)=A \hat{\zeta}(t, x)+\mathscr{F}\left[s_{\delta}(\hat{\zeta}(t))-\tilde{\mathscr{T}}[y(t)] y(t)\right](x) \\
& +\mathscr{N}_{1}[y(t)](x)+\mathscr{N}_{2}\left[y(t), \hat{\zeta}_{2}(t)\right](x)-\Theta K(y(t, x)-C \hat{\zeta}(t, x)) \\
& \hat{\zeta}(t, 0)=\mathscr{H}\left(s_{\delta}(\hat{\zeta}(t, L))-\tilde{\mathscr{T}}[y(t)] y(t)(L)\right)+\mathscr{N}_{3}[y(t)](0)
\end{aligned}
$$

with initial condition $\hat{\zeta}^{0}(x):=\hat{\zeta}(0, x)$ (for a function $\hat{\zeta}^{0}$ in $\mathscr{X}$ ), where again, as in the previous section, $\Theta$ is the diagonal matrix containing the increasing powers of the high-gain constant $\theta>1$ as in (10), $s_{\delta}$ is a saturating function satisfying the properties of items 1-4 as in Subsection 3.1 and $K$ is a constant vector gain rendering matrix $A+K C$ Hurwitz, similarly as in the previous section.

In the next subsection, we determine the transformation $\mathscr{T}$ and we show the solvability of the H-GODP.

\subsection{Indirect Solvability of the H-GODP}

We are now in a position to state the main result of this section, which includes both the existence of an infinite-dimensional transformation (14) and the convergence of observer (16) to the transformed system (T), implying that, inverting the observer state via $\mathscr{T}$, we eventually establish converge to the actual state $\xi$. This leads to an indirect solvability of the observer design problem.

Theorem 2. Assume that Assumption 2 holds for initial condition $\xi^{0} \in \mathscr{M}$. Then, the $H$-GODP is solvable for system (1a)-(2), with output (1b) and $n, q \in\{2,3\}$ by $\mathscr{T}^{-1}[y] \hat{\zeta}$ (where $\hat{\zeta}$ is the unique solution to (16)), for $\theta>1$ as a high gain and initial condition $\mathscr{T}^{-1}[y(0)] \hat{\zeta}^{0}(x)$, with $\hat{\zeta}^{0}$ satisfying zero-order and one-order compatibility conditions. More precisely, for every $\kappa>0$, there exists $\theta_{0} \geq 1$, such that for every $\theta>\theta_{0}$, the following holds for all $t \geq 0$ :

$$
\left\|\mathscr{T}^{-1}[y(t)] \hat{\zeta}(t)(\cdot)-\xi(t, \cdot)\right\|_{2-q_{0}} \leq \ell e^{-\kappa t}\left\|\mathscr{T}^{-1}[y(0)] \hat{\zeta}^{0}(\cdot)-\xi^{0}(\cdot)\right\|_{\mathscr{X}},
$$

with $\ell>0$ a polynomial in $\theta$.

We note here that in the study of internal controllability for underactuated systems, the phenomenon of loss of derivatives appeares, as the regularity of the dynamics is stronger than the regularity of the control laws, whenever the velocities are distinct (see [1, Theorem 3.1]). In the present framework aiming at the solvability of the H-GODP, we note that for $n=q=3$, the regularity of systems dynamics needs to be stronger (of order $q_{0}=2$ ) than the regularity of the space in the norm of which the asymptotic convergence of the observer is exhibited (sup-norm).

The above theorem constitutes a generalization of our previous works, see for instance [19], where the case of linear hyperbolic and semilinear parabolic systems 
via a linear state transformation is treated. We introduce here a transformation-based approach, inspired by these works, but using a nonlinear state transformation, in order to deal with the quasilinearity of the system.

Proof. Let us choose $\tilde{\mathscr{T}}$ in (14), (15) by

$$
\tilde{\mathscr{T}}\left[\xi_{1}\right]:=\left\{\begin{array}{cc}
0, & \text { when } n=2, \\
\left(\begin{array}{c}
0 \\
\tau\left[\xi_{1}\right] \\
0
\end{array}\right) \mathrm{d}_{x}, \text { when } n=3
\end{array} ;\right.
$$

where $\tau\left[\xi_{1}\right]:=\lambda_{2}\left[\xi_{1}\right]-\lambda_{3}\left[\xi_{1}\right]$.

Obviously, transformation $\mathscr{T}$, with $\tilde{\mathscr{T}}$ given as above, meets the specifications of the previous subsection independently of boundary conditions and its inverse is given by

$$
\mathscr{T}^{-1}[\cdot]=I_{n}-\tilde{\mathscr{T}}[\cdot] C .
$$

Applying the transformation chosen above to system (1a)-(2), we obtain target system $(\mathrm{T})$ of the previous subsection, with

$$
\begin{aligned}
& \mathscr{N}_{1}\left[\zeta_{1}\right]:=\left\{\begin{array}{cc}
\left(\begin{array}{cc}
\left(\lambda_{2}\left[\zeta_{1}\right]-\lambda_{1}\left[\zeta_{1}\right]\right) \partial_{x} \zeta_{1} \\
0 \\
\left(\lambda_{3}\left[\zeta_{1}\right]-\lambda_{1}\left[\zeta_{1}\right]-\tau\left[\zeta_{1}\right]\right) \partial_{x} \zeta_{1}
\end{array}\right. & n=2, \\
\left(\begin{array}{c}
\left\langle D \tau\left[\zeta_{1}\right],-\left(\lambda_{1}\left[\zeta_{1}\right]+\tau\left[\zeta_{1}\right]\right) \partial_{x} \zeta_{1}+\mathscr{F}_{1}\left[\zeta_{1}\right]\right\rangle \partial_{x} \zeta_{1}-\tau\left[\zeta_{1}\right] \\
\times \mathrm{d}_{x}\left(\lambda_{1}\left[\zeta_{1}\right] \partial_{x} \zeta_{1}-\mathscr{F}_{1}\left[\zeta_{1}\right]\right)+\lambda_{3}\left[\zeta_{1}\right] \mathrm{d}_{x}\left(\tau\left[\zeta_{1}\right] \partial_{x} \zeta_{1}\right)
\end{array}\right) & 0
\end{array}\right), n=3, \\
& \mathscr{N}_{2}\left[\zeta_{1}, \zeta_{2}\right]:= \begin{cases}0, & n=2, \\
\left(\begin{array}{cc}
\left\langle D\left[\zeta_{1}\right], \zeta_{2}\right\rangle \partial_{x} \zeta_{1} \\
0
\end{array}\right), & n=3,\end{cases} \\
& \mathscr{N}_{3}\left[\zeta_{1}\right]:=\left\{\begin{array}{cc}
0, & n=2, \\
\left(\tau\left[\zeta_{1}\right] \partial_{x} \zeta_{1}\right. \\
0
\end{array}\right), n=3,
\end{aligned}
$$

We are now in a position to prove that solutions to observer (7) converge exponentially to the solutions to transfromed system $(\mathrm{T})$. First, the well-posedness of the observer system, i.e., the global existence of unique classical solutions of regularity $C^{1}$, follows from classical arguments that one can find for instance in [3] or in our previous works [21] (details are left for the reader) and relies on Assumption 2 on existence and boundedness in the $C^{q_{0}}$ of system solutions and also on the fact that observer nonlinearities are globally Lipschitz. In this way, we now focus on the proof of the stability analysis.

Let us consider observer error by

$$
\varepsilon:=\Theta^{-1}(\hat{\zeta}-\zeta)
$$

which satisfies the following hyperbolic equations 


$$
\begin{aligned}
\varepsilon_{t}(t, x)+\lambda_{n}[y(t)](x) \varepsilon_{x}(t, x)= & \theta(A+K C) \varepsilon(t, x) \\
& +\Theta^{-1}\left(\Delta_{z(t)}[\mathscr{F}](\zeta(t)-\tilde{\mathscr{T}}[y(t)] y(t))(x)\right. \\
& \left.+\mathscr{N}_{2}\left[y(t), \hat{\zeta}_{2}(t)-\zeta_{2}(t)\right](x)\right), \\
\varepsilon(t, 0)= & \Theta^{-1} \Delta_{z(t)}[\mathscr{H}](\zeta(t)-\tilde{\mathscr{T}}[y(t)] y(t))(L),
\end{aligned}
$$

where $z:=s_{\delta}(\hat{\zeta})-\tilde{\mathscr{T}}[y] y$.

For the proof of the exponential stability of solutions to (18), the reader can refer to the Appendix of the present chapter. Following the proof in the Appendix, where an appropriate Lyapunov functional is chosen, we obtain an exponential stability result for the transformed system in the 1-norm as follows

$$
\|\hat{\zeta}(t, \cdot)-\zeta(t, \cdot)\|_{1} \leq \bar{\ell} e^{-\kappa t}\left\|\hat{\zeta}^{0}(\cdot)-\zeta^{0}(\cdot)\right\|_{1},
$$

where $\kappa>0$ is adjustable by choosing the high-gain constant $\theta$ large enough, and $\bar{\ell}>0$ is a polynomial in $\theta$.

Now, to return to the original coordinates, we notice that $\mathscr{T}[C \xi]: \mathscr{X} \mapsto \mathscr{X}$ is bounded for $\xi \in \mathscr{B}_{\delta}^{q_{0}}, \mathscr{X}$ is continuously embedded in $C^{1}\left([0, L] ; \mathbb{R}^{n}\right)$, also the extension of $\mathscr{T}^{-1}[C \xi]$ on $\left.C^{0}[0, L] ; \mathbb{R}^{n}\right)$ for $\xi \in \mathscr{B}_{\delta}^{q_{0}}$ is bounded in $\left.C^{0}[0, L] ; \mathbb{R}^{n}\right)$, and $C^{1}\left([0, L] ; \mathbb{R}^{n}\right)$ is continuously embedded in $C^{0}\left([0, L] ; \mathbb{R}^{n}\right)$. Thereby, by (19), we can calculate a constant $\ell$, polynomial again in $\theta$, such that (17) is satisfied.

The proof of Theorem 2 is complete.

Remark 2. Although in this section we considered a reduced number of states (up to 3 ), as the presence of increased number of distinct velocities imposes extra difficulties to the problem, we note that the H-GODP is solvable even for more states, but if we drop system's nonlinearity, so with the restriction that system is linear, and also space $L$-periodic. In this case, we consider a state transformation that includes higher-order differentiations in its domain than the ones in this section and, to determine it, we solve an operator Sylvester equation. We have included this generalization in previous works, see [19], where some links with problems of controllability of coupled hyperbolic PDEs as in [1] were revealed.

\section{Conclusion}

Solutions to a high-gain observer design problem for a class of quasilinear hyperbolic systems, containing also nonlocal source terms and velocities, and written in a triangular form, were presented in this chapter. A part of the state was considered as measurement. First, this problem was solved for systems with $n$ equations and only one velocity, as a direct extension of the finite-dimensional approach. Then, sufficient conditions were provided for the solvability of such a problem for the case of 2 or 3 distinct velocities. This required the introduction of a nonlinear infinitedimensional state transformation, which led to the injection of output spatial deriva- 
tives in the observer dynamics. The extension of this methodology to wider classes of infinite-dimensional systems, ISS properties of such observers, and the investigation of output feedback laws via such observers with applications to real systems, will be topics of our future works.

\section{Appendix}

\section{Observer Convergence Proofs}

In this section, we prove the Lyapunov stability part of both Theorems 1 and 2, appearing in Subsections 3.2 and 4.2, respectively.

Particularly, observer error systems appearing in Theorems 1 and 2 are given by (13) and (18), respectively. We prove here the Lyapunov stability result for error system (18) in Theorem 2 only, which is more complicated. Then, the Lyapunov stability in Theorem 1 follows, as error system (13) therein is a simpler version of (18), with $\zeta$ substituted by $\xi, \hat{\zeta}$ substituted by $\hat{\xi}, \lambda_{n}$ substituted by $\lambda$, and $\tilde{\mathscr{T}}=$ $0, \mathscr{N}_{2}=0$.

To prove the exponential stability of the solution to error system (18) at the origin in Theorem 2 , let us define a Lyapunov functional $\mathscr{W}_{p}: C^{1}\left([0, L] ; \mathbb{R}^{n}\right) \rightarrow \mathbb{R}$ by

$$
\begin{aligned}
\mathscr{W}_{p}[\varepsilon] & :=\left(\int_{0}^{L} \pi(x) \exp \left(p \mu_{\theta, \delta} x\right) \mathscr{G}_{p}[\varepsilon](x) \mathrm{d} x\right)^{1 / p} ; \\
\mathscr{G}_{p}[\varepsilon] & :=\left(\varepsilon^{\top} P \varepsilon+\rho_{0} \varepsilon_{t}^{\top} P \varepsilon_{t}\right)^{p},
\end{aligned}
$$

where $\varepsilon:=\Theta^{-1}(\hat{\zeta}-\zeta)$ is the observer error for the transformed via $\mathscr{T}$ system, $\rho_{0} \in(0,1)$ is a constant (to be chosen appropriately), $p \in \mathbb{N}, P \in \mathbb{R}^{n \times n}$ is positive definite and symmetric, satisfying (11), $\pi:[0, L] \rightarrow \mathbb{R}$ is given by

$$
\pi(x):=(\bar{\pi}-1) \frac{x}{L}+1 ; \bar{\pi}:=\frac{\sup _{\|\zeta\|_{0} \leq \delta} \lambda_{n}[C \zeta]}{\inf _{\|\zeta\|_{0} \leq \delta} \lambda_{n}[C \zeta]},
$$

with $\pi(x) \in[1, \bar{\pi}]$, and constant $\mu_{\theta, \delta}$ is given by

$$
\mu_{\theta, \delta}:=\frac{1}{L} \ln \left(\mu_{\delta} \theta^{2 n-2}\right)
$$

where 


$$
\begin{aligned}
\mu_{\delta}:= & \frac{|P|}{\underline{\operatorname{eig}(P)}} \max \left\{\gamma_{1, \delta}^{2}, \gamma_{2, \delta}^{2}, \gamma_{3, \delta}^{2} \delta_{1}^{2}, \gamma_{1, \delta} \gamma_{2, \delta} \delta_{1}\right\} ; \\
\gamma_{1, \delta}:= & \sup _{|\zeta(L)| \leq \delta, y \in C^{1}([0, L] ; \mathbb{R}), \hat{\zeta}(L) \in \mathbb{R}^{n}, \mathcal{\varepsilon}(L) \neq 0} \frac{\left|\Theta^{-1} \Delta_{z}[\mathscr{H}](\zeta-\tilde{\mathscr{T}}[y] y)(L)\right|}{\theta^{n-1}|\mathcal{\varepsilon}(L)|}, \\
\gamma_{2, \delta}:= & \theta^{1-n} \sup _{\hat{\zeta}(L) \in \mathbb{R}^{n},\|y\|_{1} \leq \delta}\left|\Theta^{-1} D \mathscr{H}[z](L) D s_{\delta}(\hat{\zeta}(L)) \Theta\right|, \\
\gamma_{3, \delta}:= & \sup _{|\zeta(L)| \leq \delta, \hat{\zeta}(L) \in \mathbb{R}^{n},\|y\|_{1} \leq \delta, \varepsilon(L) \neq 0} \frac{1}{\theta^{n-1}|\mathcal{E}(L)|} \\
& \times\left|\Theta^{-1}\left(\Delta_{z}[D \mathscr{H}](\zeta-\tilde{\mathscr{T}}[y] y)(L) D s_{\delta}(\hat{\zeta})+D \mathscr{H}(z(L)) \Delta_{\hat{\zeta}}\left[D s_{\delta}\right](\zeta)(L)\right)\right|,
\end{aligned}
$$

with

$$
z:=s_{\delta}(\hat{\zeta})-\tilde{T}[y] y
$$

and also

$$
\begin{aligned}
\delta_{1} & :=\sup _{\zeta \in \mathscr{B}_{\delta}^{1},\|y\|_{q_{0}} \leq \delta}\left(\left\|\zeta_{t}\right\|_{0}+\left\|d_{t}(\tilde{\mathscr{T}}[y] y)\right\|_{0}\right) \\
& =\sup _{\xi \in \mathscr{B}_{\delta}^{q_{0}}}\left(\left\|(\mathscr{T}[C \xi] \xi)_{t}\right\|_{0}+\left\|d_{t}(\tilde{\mathscr{T}}[C \xi] C \xi)\right\|_{0}\right),
\end{aligned}
$$

which is proven to be finite after substituting $\xi_{t}$ by $-\Lambda[C \xi] \xi_{x}+A \xi+\mathscr{F}[\xi]$ by (1a) and invoking the regularity of $\Lambda$, and the boundedness of the mapping $\mathscr{T}$ in $\mathscr{X}$.

Note here that the above constants with subscript $\delta$ do not have any dependence on the observer gain $\theta$ but on the global bound $\delta$ of system solutions (coming from Assumption 2), otherwise such a dependence will be explicit by use of a subscript $\theta$. We took also into account the implication

$$
\xi \in \mathscr{B}_{\delta}^{q_{0}} \text { (coming from Assumption 2) } \Rightarrow \mathscr{T}[C \xi] \xi=: \zeta \in \mathscr{B}_{\delta}^{1} .
$$

By invoking global existence of solutions to observer system (16) and Assumption 2, which establishes global unique classical solutions for system (1a)(2), and boundedness of mapping $\mathscr{T}$ in $\mathscr{X}$, we are now in a position to define $G_{p}, W_{p}:[0,+\infty) \rightarrow \mathbb{R}$

$$
G_{p}(t):=\mathscr{G}_{p}[\varepsilon](t), W_{p}(t):=\mathscr{W}_{p}[\varepsilon](t), \forall t \geq 0 .
$$

Before taking the time-derivative of the Lyapunov function, we temporarily assume that $\varepsilon$ is of class $C^{2}$ and we can, thus, derive the hyperbolic equations satisfied by $\varepsilon_{t}$ (details are left to the reader). Calculating the time-derivative $\dot{W}_{p}$ along the classical solutions of the hyperbolic equations (18) for $\varepsilon$ and of the corresponding hyperbolic equations for $\varepsilon_{t}$, we get 


$$
\begin{aligned}
& \dot{W}_{p}=\frac{1}{p} W_{p}^{1-p} \int_{0}^{L} p \pi(x) \exp \left(p \mu_{\theta, \delta} x\right) G_{p-1}(x) \\
& \times\left(\varepsilon_{t}(x)^{\top} P \varepsilon(x)+\varepsilon^{\top}(x) P \varepsilon_{t}(x)+\rho_{0} \varepsilon_{t t}^{\top}(x) P \varepsilon_{t}(x)+\rho_{0} \varepsilon_{t}(x)^{\top} P \varepsilon_{t t}(x)\right) \mathrm{d} x,
\end{aligned}
$$

where after substituting dynamics of $\varepsilon, \varepsilon_{t}$ and performing integration by parts, we obtain

$$
\dot{W}_{p}=W_{p}^{1-p}\left(\frac{1}{p} T_{1, p}+\frac{1}{p} T_{2, p}+T_{3, p}+T_{4, p}\right),
$$

where

$$
\begin{aligned}
T_{1, p}:= & -\pi(L) \lambda_{n}[y](L) \exp \left(p \mu_{\theta, \delta} L\right) G_{p}(L)+\pi(0) \lambda_{n}[y](0) G_{p}(0), \\
T_{2, p}:= & \int_{0}^{L} \mathrm{~d} x\left[\pi(x) \lambda_{n}[y](x) \exp \left(p \mu_{\theta, \delta} x\right)\right] G_{p}(x) \mathrm{d} x, \\
T_{3, p}:= & 2 \int_{0}^{L} \pi(x) \exp \left(p \mu_{\theta, \delta} x\right) G_{p-1}(x)\left[\varepsilon^{\top}(x) P \Theta^{-1}\right. \\
& \times\left(\Delta_{z}[\mathscr{F}](\zeta-\tilde{T}[y] y)(x)+\mathscr{N}_{2}\left[y, \hat{\zeta}_{2}-\zeta_{2}\right](x)\right) \\
& +\rho_{0} \varepsilon_{t}^{\top}(x) \operatorname{Sym}(P \mathscr{K}[\zeta](x)) \varepsilon_{t}(x)+\rho_{0} \varepsilon_{t}^{\top}(x) P\left(\mathscr{K}^{\hat{\zeta}}[\zeta](x)\right. \\
& \left.\left.+\Theta^{-1}\left\langle D \mathscr{F}[z], D s_{\delta}(\hat{\zeta}) \Theta \varepsilon_{t}\right\rangle(x)+\Theta^{-1} d_{t}\left(\mathscr{N}_{2}\left[y, \hat{\zeta}_{2}-\zeta_{2}\right](x)\right)\right)\right] \mathrm{d} x, \\
T_{4, p}:= & \theta \int_{0}^{L} \pi(x) \exp \left(p \mu_{\theta, \delta} x\right) G_{p-1}(x) \\
& \times\left[2 \varepsilon^{\top} \operatorname{Sym}(P(A+K C)) \varepsilon+2 \rho_{0} \varepsilon_{t}^{\top} \operatorname{Sym}(P(A+K C)) \varepsilon_{t}\right. \\
& \left.-\rho_{0} \varepsilon_{t}^{\top} P \mathscr{K}[\zeta](A+K C) \varepsilon-\rho_{0} \varepsilon^{\top}(A+K C)^{\top} \mathscr{K}^{\top}[\zeta] P \varepsilon_{t}\right] \mathrm{d} x,
\end{aligned}
$$

where $\mathscr{K}: \mathscr{B}_{\delta}^{1} \rightarrow C^{0}\left([0, L] ; \mathbb{R}^{n \times n}\right)$ is a bounded mapping defined by

$$
\mathscr{K}[\zeta]:=\left(\lambda_{n}\left[\zeta_{1}\right]\right)^{-1}\left\langle D \lambda_{n}\left[\zeta_{1}\right], C \zeta_{t}\right\rangle I_{n}
$$

and $\mathscr{K}^{\hat{\zeta}}: \mathscr{B}_{\delta}^{1} \rightarrow C^{0}\left([0, L] ; \mathbb{R}^{n}\right)$, parametrized by $\hat{\zeta} \in C^{0}\left([0, L] ; \mathbb{R}^{n}\right)$, is given by

$$
\begin{aligned}
\mathscr{K}^{\hat{\zeta}}[\zeta]:= & -\mathscr{K}[\zeta] \Theta^{-1}\left(\Delta_{z}[\mathscr{F}]\left(\zeta-\tilde{\mathscr{T}}\left[\zeta_{1}\right] \zeta_{1}\right)+\mathscr{N}_{2}\left[\zeta_{1}, \hat{\zeta}_{2}-\zeta_{2}\right]\right) \\
& +\Theta^{-1}\left(\left\langle\Delta_{z}[D \mathscr{F}]\left(\zeta-\tilde{\mathscr{T}}\left[\zeta_{1}\right] \zeta_{1}\right), \zeta_{t}-d_{t}\left(\tilde{\mathscr{T}}\left[\zeta_{1}\right] \zeta_{1}\right)\right\rangle\right. \\
& \left.+\left\langle D \mathscr{F}[z], \Delta_{\hat{\zeta}}\left[D s_{\delta}\right](\zeta) \zeta_{t}\right\rangle\right) .
\end{aligned}
$$

After substituting boundary conditions for $\varepsilon, \varepsilon_{t}$ in $T_{1, p}$ and by virtue of (21) and (22b), we obtain the following inequality

$$
T_{1, p} \leq \sup _{\zeta \in \mathscr{B}{ }_{\delta}^{1}}\left(\lambda_{n}[C \zeta]\right) G_{p}(L)\left(-\exp \left(p \mu_{\theta, \delta} L\right)+\left(\theta^{2 n-2} \mu_{\delta}\right)^{p}\right)
$$


and, subsequently, by (22a), we get

$$
T_{1, p} \leq 0
$$

For $T_{2, p}$, we can easily derive the following bound

$$
T_{2, p} \leq\left(\omega_{1, \delta}+p\left|\mu_{\theta, \delta}\right| \omega_{2, \delta}\right) W_{p}^{p}
$$

where

$$
\omega_{1, \delta}:=\frac{|P| \delta \sup _{\zeta \in \mathscr{B}_{\delta}^{1}}\left|\mathrm{~d}_{x} \lambda_{n}[C \zeta]\right|}{\underline{\operatorname{eig}}(P)}, \quad \omega_{2, \delta}:=\frac{|P| \sup _{\zeta \in \mathscr{B}_{\delta}^{1}} \lambda_{n}[C \zeta]}{\underline{\operatorname{eig}(P)}} .
$$

By taking into account that the dynamics are locally Lipschitz, we obtain

$$
T_{3, p} \leq \omega_{3, \delta}\left\|G_{1}(\cdot)\right\|_{0} W_{p-1}^{p-1}
$$

where

$$
\begin{aligned}
\omega_{3, \delta}:= & 2 \frac{|P|}{\underline{\operatorname{eig}(P)}} \max \left\{\gamma_{4, \delta}, \gamma_{5, \delta}, \gamma_{6, \delta}, \sup _{\zeta \in \mathscr{B}_{\delta}^{1}} \mid \mathscr{K}[\zeta], \frac{3}{2} \gamma_{7, \delta}\right\} ; \\
\gamma_{4, \delta}:= & \sup _{\zeta \in \mathscr{B}_{\delta}^{1}, y \in C^{1}([0, L] ; \mathbb{R}), \hat{\zeta} \in C^{0}\left([0, L] ; \mathbb{R}^{n}\right), \varepsilon \neq 0} \frac{1}{\|\varepsilon\|_{0}} \\
& \times\left\|\Theta^{-1}\left(\Delta_{z}[\mathscr{F}](\zeta-\tilde{\mathscr{T}}[y] y)+\mathscr{N}_{2}\left[y, \hat{\zeta}_{2}-\zeta_{2}\right]\right)\right\|_{0}, \\
\gamma_{5, \delta}:= & \sup _{\zeta \in \mathscr{B}_{\delta}^{1}, \hat{\zeta} \in C^{0}\left([0, L] ; \mathbb{R}^{n}\right), \varepsilon \neq 0} \frac{\left\|\mathscr{K}^{\hat{\zeta}}[\zeta]\right\|_{0}}{\|\varepsilon\|_{0}}, \\
\gamma_{6, \delta}:= & \sup _{\hat{\zeta} \in C^{0}\left([0, L] ; \mathbb{R}^{n}\right),\|y\|_{1} \leq \delta, \varepsilon_{t} \in C^{0}\left([0, L] ; \mathbb{R}^{n}\right), \varepsilon_{t} \neq 0} \frac{\left\|\Theta^{-1}\left\langle D \mathscr{F}[z], D s_{\delta}(\hat{\zeta}) \Theta \varepsilon_{t}\right\rangle\right\|_{0}}{\left\|\varepsilon_{t}\right\|_{0}}, \\
\gamma_{7, \delta}:= & \sup _{\zeta \in \mathscr{B}_{\delta}^{1},\|y\|_{q_{0}} \leq \delta, \hat{\zeta}_{2} \in C^{0}([0, L] ; \mathbb{R}), \varepsilon, \varepsilon_{t} \neq 0} \frac{\left\|\Theta^{-1} d_{t}\left(\mathscr{N}_{2}\left[y, \hat{\zeta}_{2}-\zeta_{2}\right]\right)\right\|_{0}}{\|\varepsilon\|_{0}+\left\|\varepsilon_{t}\right\|_{0}},
\end{aligned}
$$

where we can easily see that $\gamma_{7, \delta}$ is finite, since quantity $d_{t}\left(\mathscr{N}_{2}\left[y, \hat{\zeta}_{2}-\zeta_{2}\right]\right)$ is given by

$$
\begin{aligned}
d_{t}\left(\mathscr{N}_{2}\left[y, \hat{\zeta}_{2}-\zeta_{2}\right]\right)= & \mathscr{N}_{2}\left[y, \theta^{2} \partial_{t} \varepsilon_{2}\right] \\
& +\left(\begin{array}{c}
0 \\
y_{t x}\left\langle D \tau[y], \theta^{2} \varepsilon_{2}\right\rangle \\
0
\end{array}\right)+\left(\begin{array}{c}
0 \\
y_{x}\left\langle D^{2} \tau[y], \theta^{2} y_{t} \varepsilon_{2}\right\rangle \\
0
\end{array}\right)
\end{aligned}
$$


and $y_{t}, y_{t x}$ are uniformly bounded, whenever $\xi \in \mathscr{B}_{\delta}^{q_{0}}$ (see Assumption 2), as seen by hyperbolic dynamics (1a).

Term $T_{4, p}$, which will lead to the negativity of the Lyapunov derivative, can be rewritten in the following way

$$
T_{4, p}:=-\theta \int_{0}^{L} \pi(x) \exp \left(p \mu_{\theta, \delta} x\right) G_{p-1}(x) E^{\top}(x) \Sigma[\zeta](x) E(x) \mathrm{d} x,
$$

where $E:=\left(\varepsilon \varepsilon_{t}\right)^{\top}$ and after utilizing (11), $\Sigma: \mathscr{B}_{\delta}^{1} \rightarrow C^{0}\left([0, L] ; \mathbb{R}^{2 n \times 2 n}\right)$ is given by

$$
\Sigma[\zeta]:=\left(\begin{array}{cc}
I_{n} & -\rho_{0}(A+K C)^{\top} \mathscr{K}^{\top}[\zeta] P \\
-\rho_{0} P \mathscr{K}[\zeta](A+K C) & \rho_{0} I_{n}
\end{array}\right) .
$$

Now, we can easily verify (using Schur complement) that for all $w \in \mathbb{R}^{2 n} \backslash 0$, we have $\inf _{\zeta \in \mathscr{B}_{\delta}^{1}} \frac{w^{\top} \Sigma[\zeta] w}{|w|^{2}}>0$, if

$$
\rho_{0}<\min \left\{\frac{1}{|P|^{2}|A+K C|^{2}\left(\sup _{\zeta \in \mathscr{B}_{\delta}^{1}} \mathscr{K}[\zeta]\right)^{2}}, 1\right\}
$$

It turns out that for every choice of matrices $P$ and $K$ satisfying (11), there always exists a $\rho_{0}$, such that (6) is satisfied and this fact renders $\Sigma$ positive. Consequently, there exists $\sigma_{\delta}>0$, such that

$$
T_{4, p} \leq-\theta \frac{\sigma_{\delta}}{|P|} W_{p}^{p}
$$

We note here that all the previously defined constants with subscript $\delta$ (for instance $\left.\gamma_{i, \delta}, i=4, \ldots, 7\right)$ have no dependence on the observer gain constant $\theta$ and this is a consequence of the triangularity of the involved nonlinear mappings, similarly as in the classical high-gain observer designs [13]. This property turns out to be sufficient for the solvability of the H-GODP. More precisely, while bounding the Lyapunov derivative from above, the independence of these parameters on $\theta$ shall not add positive terms with linear (or higher-order) dependence on $\theta$. On the other hand, negative terms will appear depending linearly on $\theta$ as a direct consequence of the assumed observability of the pair $(A, C)$. This will render the negativity of the Lyapunov derivative feasible.

Now, combining (28), (29), (30), and (31) with (24), we obtain

$$
\dot{W}_{p} \leq\left(-\theta \omega_{4, \delta}+\omega_{5, \delta} \ln (\theta)+\omega_{6, \delta}\right) W_{p}+\omega_{3, \delta} W_{p}^{1-p} W_{p-1}^{p-1}\left\|G_{1}(\cdot)\right\|_{0},
$$

where $\omega_{4, \delta}:=\frac{\sigma_{\delta}}{|P|}, \omega_{5, \delta}:=\frac{\omega_{2, \delta}(2 n-2)}{L}, \omega_{6, \delta}:=\frac{\omega_{2, \delta}}{L}\left|\ln \mu_{\delta}\right|$. Now, using Hölder's inequality, one can obtain 


$$
W_{p-1}^{p-1} \leq W_{p}^{p-1}\|\pi(\cdot)\|_{0}^{1 / p} .
$$

Utilizing the above inequality, (32) gives

$$
\dot{W}_{p} \leq\left(-\theta \omega_{4, \delta}+\omega_{5, \delta} \ln (\theta)+\omega_{6, \delta}\right) W_{p}+\omega_{3, \delta} \bar{\pi}^{1 / p}\left\|G_{1}(\cdot)\right\|_{0} .
$$

We obtained the estimate (33) of $\dot{W}_{p}$ for $\varepsilon$ of class $C^{2}$, but, by invoking density arguments, the results remain valid with $\varepsilon$ only of class $C^{1}$ (see [9] for further details on analogous statements). Taking the limit as $p \rightarrow+\infty$ of both sides od (33), we get in the distribution sense in $(0,+\infty)$,

$$
\frac{\mathrm{d}}{\mathrm{d} t}\left\|G_{1}(t, \cdot)\right\|_{0} \leq\left(-\theta \omega_{4, \delta}+\omega_{5, \delta} \ln (\theta)+\omega_{7, \delta}\right)\left\|G_{1}(t, \cdot)\right\|_{0},
$$

where $\omega_{7, \delta}:=\omega_{3, \delta}+\omega_{6, \delta}$.

Now, one can select the high gain $\theta$, such that $\theta>\theta_{0}$, where $\theta_{0} \geq 1$ is such that

$$
-\theta \omega_{4, \delta}+\omega_{5, \delta} \ln (\theta)+\omega_{7, \delta} \leq-2 \kappa_{\delta}, \forall \theta>\theta_{0}
$$

for some $\kappa_{\delta}>0$. One can easily check that for any $\kappa_{\delta}>0$, there always exists a $\theta_{0} \geq 1$, dependent on the involved constants, such that the previous inequality is satisfied.

Subsequently, (34) yields to the following differential inequality in the distribution sense in $(0,+\infty)$

$$
\frac{\mathrm{d}}{\mathrm{d} t}\left\|G_{1}(t, \cdot)\right\|_{0} \leq-2 \kappa_{\delta}\left\|G_{1}(t, \cdot)\right\|_{0}
$$

and by the comparison lemma, we get

$$
\left\|G_{1}(t, \cdot)\right\|_{0} \leq e^{-2 \kappa_{\delta} t}\left\|G_{1}(0, \cdot)\right\|_{0}, \forall t \geq 0 .
$$

Now, by the dynamics (18) we can obtain the following inequality

$$
\inf _{\zeta \in \mathscr{B}_{\delta}^{1}} \lambda_{n}[C \zeta]\left\|\varepsilon_{x}\right\|_{0}-s_{\delta, \theta}\|\varepsilon\|_{0} \leq\left\|\varepsilon_{t}\right\|_{0} \leq \sup _{\zeta \in \mathscr{B}_{\delta}^{1}} \lambda_{n}[C \zeta]\left\|\varepsilon_{x}\right\|_{0}+s_{\delta, \theta}\|\varepsilon\|_{0}
$$

where $s_{\delta, \theta}:=\theta|A+K C|+\gamma_{4, \delta}$. Invoking these inequalites, also (22a), estimate (36) and the following inequality,

$$
\frac{\rho_{0}}{2} e^{\frac{\mu_{\theta, \delta^{-}}\left|\mu_{\theta, \delta}\right|}{2}} \underline{\operatorname{eig}}(P)\left(\|\varepsilon\|_{0}+\left\|\varepsilon_{t}\right\|_{0}\right)^{2} \leq\left\|G_{1}(\cdot)\right\|_{0} \leq e^{\frac{\mu_{\theta, \delta}+\left|\mu_{\theta, \delta}\right|}{2}} L|P|\left(\|\varepsilon\|_{0}+\left\|\varepsilon_{t}\right\|_{0}\right)^{2}
$$

we obtain

$$
\|\varepsilon\|_{1} \leq \ell_{\theta, \delta} e^{-\kappa_{\delta} t}\left\|\varepsilon^{0}\right\|_{1}, \forall t \geq 0,
$$

where $\varepsilon^{0}(x):=\varepsilon(0, x)$ and 


$$
\begin{aligned}
\ell_{\theta, \delta}:= & \sqrt{\frac{|P|}{\rho_{0} \underline{\operatorname{eig}(P)}}}\left(\mu_{\delta}\right)^{\frac{1}{2 L}} \theta^{\frac{n-1}{L}} \\
& \times \max \left\{s_{\delta, \theta}+1, \frac{1}{\inf _{\zeta \in \mathscr{B}_{\delta}^{1}} \lambda_{n}[C \zeta]}\right\} \max \left\{1+2 s_{\delta, \theta}, 2 \sup _{\zeta \in \mathscr{B}_{\delta}^{1}} \lambda_{n}[C \zeta]\right\} .
\end{aligned}
$$

By (37), we derive the following estimate, which holds for every $t \geq 0$

$$
\|\hat{\zeta}(t, \cdot)-\zeta(t, \cdot)\|_{1} \leq \bar{\ell}_{\theta, \delta} e^{-\kappa_{\delta} t}\left\|\hat{\zeta}^{0}-\zeta^{0}\right\|_{1}
$$

where $\bar{\ell}_{\theta, \delta}:=\theta^{n-1} \ell_{\theta, \delta}$.

Note that the polynomial dependence of $\bar{\ell}_{\delta, \theta}$ on $\theta$ is a phenomenon appearing also in high-gain observer designs in finite dimensions.

The proof of the exponential convergence of $\hat{\zeta}$ to $\zeta$ in the 1-norm is complete.

\section{References}

1. Alabau-Boussouira F., Coron J.-M., and Olive G.: Internal controllability of first order quasilinear hyperbolic systems with a reduced number of controls. SIAM Journal on Control and Optimization 55(1), 300-323 (2017)

2. Anfinsen H., Diagne M., Aamo O. M., Krstić M.: An adaptive observer design for $n+1$ coupled linear hyperbolic PDEs based on swapping. IEEE Transactions on Automatic Control 61(12), 3979-3990 (2016)

3. Bastin G. and Coron J.-M.: Stability and boundary stabilization of 1-D hyperbolic systems. Progress in Nonlinear Differential Equations and Their Applications, Springer International Publishing (2016)

4. Besançon G: Nonlinear observers and applications. Springer Verlag, New York (2007)

5. Bounit H. and Hammouri H.: Observer design for distributed parameter dissipative bilinear systems. Appl. Math. Comput. Sci. 8, 381-402 (1998)

6. Castillo F., Witrant E., Prieur C., and Dugard L.: Boundary observers for linear and quasilinear hyperbolic systems with application to flow control. Automatica 49(11), 3180-3188 (2013)

7. Christofides P. D. and Daoutidis P.: Feedback control of hyperbolic pde systems. AIChE Journal 42(11), 3063-3086 (1996)

8. Coron J.-M., Vazquez R., Krstić M. , and Bastin G.: Local exponential stabilization of a 2x2 quasilinear hyperbolic system using backstepping. SIAM J. Control Optim. 51(3), 2005-2035 (2013)

9. Coron J.-M. and Bastin G.: Dissipative boundary conditions for one-dimensional quasi-linear hyperbolic systems: Lyapunov stability for the $C 1$-norm. SIAM Journal on Control and Optimization 53(3), 1464-1483 (2015)

10. Coron J.-M. and Nguyen H. M.: Optimal time for the controllability of linear hyperbolic systems in one dimensional space. SIAM Journal on Control and Optimization 57(2), 1127 1156 (2019)

11. Di Meglio F., Krstić M., and Vasquez R.: A backstepping boundary observer for a class of linear first-order hyperbolic systems. European Control Conf., Zurich, Switzerland, 1597$1602(2013)$

12. Gauthier J. P. and Bornard G.: Observability for any $u(t)$ of a class of nonlinear systems. IEEE Transactions on Automatic Control 26(4), 922-926 (1981) 
13. Gauthier J. P., Hammouri H., and Othman S.: A simple observer for nonlinear systems: applications to bioreactors. IEEE Transactions on Automatic Control 37(6), 875-880 (1992)

14. Hasan A., Aamo O. M., and Krstić M.: Boundary observer design for hyperbolic PDE-ODE cascade systems. Automatica 68, 75-86 (2016)

15. Karafyllis I., Ahmed-Ali T., and Giri F.: Sampled-data observers for 1-D parabolic PDEs with non-local outputs. Systems and Control Letters 133 (2019)

16. Keimer A., Pflug L., and Spinola M.: Existence, uniqueness and regularity of multidimensional nonlocal balance laws with damping. J. Math. Anal. Appl. 466, 18-55 (2018)

17. Khalil H. K.: High-gain observers in nonlinear feedback control. Advances in Design and Control, SIAM (2017)

18. Khalil H. K. and Praly L.: High-gain observers in nonlinear feedback control. International Journal of Robust and Nonlinear Control 24(6), 993-1015 (2014)

19. Kitsos C.: High-gain observer design for systems of PDEs. PhD Thesis. Univ. Grenoble Alpes (2020)

20. Kitsos C., Besançon G., and Prieur C.: High-gain observer design for a class of quasilinear integro-differential hyperbolic systems - application to an epidemic model. Conditionally accepted in IEEE Transactions on Automatic Control (2021)

21. Kitsos C., Besançon G., and Prieur C.: High-gain observer design for some semilinear reaction-diffusion systems: a transformation-based approach. IEEE Control Systems Letters 5(2), 629-634 (2021)

22. Kmit I.: Classical solvability of nonlinear initial-boundary problems for first-order hyperbolic systems. International Journal of Dynamical Systems and Differential Equations 1(3), 191195 (2008)

23. Li T-T.: Exact boundary observability for quasilinear hyperbolic systems. ESAIM: Control, Optimisation and Calculus of Variations14(4), 759-766 (2008)

24. Lissy P., and Zuazua E.: Internal observability for coupled systems of linear partial differential equations. SIAM Journal on Control and Optimization, Society for Industrial and Applied Mathematics 57(2), 832-853 (2019)

25. Meurer T.: On the extended Luenberger-type observer for semilinear distributed-parameter systems. IEEE Trans. Autom. Control 58(7), 1732-1743 (2013)

26. Nguyen V., Georges D., and Besançon G.: State and parameter estimation in 1-d hyperbolic PDEs based on an adjoint method. Automatica 67, 185-191 (2016)

27. Prieur C., Girard A., and Witrant E.: Stability of switched linear hyperbolic systems by Lyapunov techniques. IEEE Transactions on Automatic Control 59(8), 2196-2202 (2014)

28. Schaum A., Moreno J. A., Alvarez J., and Meurer T.: A simple observer scheme for a class of 1-D semi-linear parabolic distributed parameter systems, European Control Conf., Linz, Austria, 49-54 (2015)

29. Vazquez R. and Krstić M.: Boundary observer for output-feedback stabilization of thermalfluid convection loop. IEEE Trans. Control Syst. Technol. 18(4), 789-797 (2010)

30. Xu C., Ligarius P., and Gauthier J. P.: An observer for infinite-dimensional dissipative bilinear systems. Comput. Math. Appl. 29(7), 13-21 (1995) 